\title{
On the Ground or in the Air? A Methodological Experiment on Crop Residue Cover Measurement in Ethiopia
}

\author{
Frédéric Kosmowski $^{1} \cdot$ James Stevenson $^{1} \cdot \mathrm{Jeff} \mathrm{Campbel}^{2} \cdot$ Alemayehu Ambel $^{3}$. \\ Asmelash Haile Tsegay ${ }^{3}$
}

Received: 27 February 2017 / Accepted: 23 May 2017 / Published online: 8 June 2017

(c) The Author(s) 2017. This article is an open access publication

\begin{abstract}
Maintaining permanent coverage of the soil using crop residues is an important and commonly recommended practice in conservation agriculture. Measuring this practice is an essential step in improving knowledge about the adoption and impact of conservation agriculture. Different data collection methods can be implemented to capture the field level crop residue coverage for a given plot, each with its own implication on survey budget, implementation speed and respondent and interviewer burden. In this paper, six alternative methods of crop residue coverage measurement are tested among the same sample of rural households in Ethiopia. The relative accuracy of these methods are compared against a benchmark, the line-transect method. The alternative methods compared against the benchmark include: (i) interviewee (respondent) estimation; (ii) enumerator estimation visiting the field; (iii) interviewee with visual-aid without visiting the field; (iv) enumerator with visual-aid visiting the field; (v) field picture collected with a drone and analyzed with image-processing methods and (vi) satellite picture of the field analyzed with remote sensing methods. Results of the methodological experiment show that survey-based methods tend to underestimate field residue cover. When quantitative data on cover are needed,
\end{abstract}

Electronic supplementary material The online version of this article (doi:10.1007/s00267-017-0898-0) contains supplementary material, which is available to authorized users.

Frédéric Kosmowski

frederic.kosmowski@fao.org

1 CGIAR Standing Panel on Impact Assessment, Food and Agriculture Organization of the United Nations, Rome, Italy

2 Spatial Solutions Inc, Bend, OR, USA

3 The World Bank, Washington, DC, USA the best estimates are provided by visual-aid protocols. For categorical analysis (i.e., $>30 \%$ cover or not), visual-aid protocols and remote sensing methods perform equally well. Among survey-based methods, the strongest correlates of measurement errors are total farm size, field size, distance, and slope. Results deliver a ranking of measurement options that can inform survey practitioners and researchers.

Keywords Conservation agriculture adoption $\cdot$ Crop residue coverage $\cdot$ Agricultural remote sensing $\cdot$ Drone $\cdot$ NDTI

\section{Introduction}

In many parts of the world, soil degradation threatens the productive capacity of farmland while demographic pressure limits the potential to farm new lands. In order to achieve increases in agricultural productivity, a better and more sustainable use of land is advocated (sustainable intensification) by proponents of conservation agriculture. Thus, conservation agriculture has received considerable attention among scholars and policy makers (Kassam et al. 2009; Erenstein et al. 2012; Thierfelder and Wall 2012; Tesfaye et al. 2015). The CGIAR—a global partnership on international agriculture research-has invested significantly into conservation agriculture research over the last decades (Renkow and Byerlee 2010) and a growing number of development organizations have been promoting conservation agriculture, with recent efforts focusing on smallholder farming systems in sub-Saharan Africa and Asia (Stevenson et al. 2014). 
Conservation agriculture is a set of practices aimed at reducing soil erosion, improving water management and enhancing crop yields. According to the Food and Agriculture Organization (FAO) definition, conservation agriculture is characterized by three crop management practices: (i) minimum mechanical soil disturbance (zero or minimum tillage); (ii) permanent soil cover with crop residues or cover crops, and (iii) diversification of crop species grown in sequences (crop rotation) and/or associations (intercropping). These practices are interlinked but recent evidence from meta-analyses indicate that permanent soil cover is an essential component (Corbeels et al. 2014; Pittelkow et al. 2015).

Conservation agriculture has risen to prominence in the policy discourse on sustainable intensification in spite of a lack of evidence of its adoption by farmers-a problem that is particularly acute in sub-Saharan Africa (Stevenson et al. 2014). As noted by Andersson and D'Souza (2014), considerable variation exists in those adoption estimates that are available and it is not clear how many hectares of land are currently under conservation agriculture. Despite the important implications for policy and resource allocation, very few studies have examined the accuracy of alternative methods to estimate conservation agriculture adoption. One exception is the paper by Kondylis et al. (2015) who found that, in the context of a household survey in Mozambique, questions about adoption of mulching and strip tillage were answered "correctly" (when verified by visits to the plot) by between 85 and $95 \%$ of respondents, while the error rate was more pronounced for intercropping ( $80 \%$ correct).

A sufficient condition for adoption of the second pillar of conservation agriculture-crop residue coverage-is where a plot has at least $30 \%$ of the soil surface covered by organic material immediately after the planting operation. The $30 \%$ threshold is used in international agricultural statistics (OECD 2001; FAO 2016). FAO's AQUASTAT database goes even further, by distinguishing three categories of adopters: $30-60,60-90$, and $>90 \%$ ground cover.

Studies of conservation agriculture adoption have been lacking in the literature, and in order to be policy-relevant, adoption estimates should be grounded in nationally representative surveys. Throughout sub-Saharan Africa, several National Statistical Institutes collect data on crop residue use, although not always for the purpose of conservation agriculture measurement. Statistical institutes in Malawi, Niger, Tanzania, and Uganda have measured crop residue use as a binary variable. Other countries, such as Zambia, focus on the main tillage method utilized by farmers, whereas Ethiopia collects data on estimated field residue coverage. Such data are self-reported by the farmer. Assessing whether a condition of $30 \%$ minimum crop residue coverage is met could be particularly error-prone using self-reported measures. Furthermore, in the context of econometric analysis that estimates the role that adoption of conservation agriculture has on productivity and other metrics, there is a danger that self-reported measures could be biased-individuals more skilled at farming (something that is typically unmeasured in surveys) could also be more skilled at identifying their adoption status correctly. Therefore, there is a need to identify low-cost, reliable methods for capturing this key element of conservation agriculture.

Low-cost alternative approaches to crop residue cover measurement include the use of visual-aid protocols to approximate the current residue cover, as well as field images or videos analyzed through image-processing methods (Woebbeck et al. 1995). The use of remote sensing technologies has also been tested in the US, and tillage indices have been applied with mixed results (Nagler et al. 2003; Serbin et al. 2009a, b; Daughtry et al. 2010). Although several challenges remain (Zheng et al. 2014), remote sensing technology could represent a huge step forward, by allowing broad-scale mapping of conservation agriculture adoption.

This data capture experiment contributes to a growing literature on agricultural survey methodology (Carletto et al. 2013; Zezza et al. 2016). In order to assess the accuracy of different measurement methods, real survey conditions should be reproduced in an experimental setting. This can be achieved by using a clear benchmark or reference method against which all other methods are compared. In this experiment, a within-plot line-transect benchmark is compared to six lower-cost, alternative methods for estimating crop residue coverage for a plot. The data collection methods under consideration should be able to match the reference distribution of crop residues on the plot as determined by the line transect (LT). ${ }^{1}$

Two hypothesis are explored in this paper. The first expectation is that methods relying on a self-reported estimations fail to capture the indicator of interest. We therefore hypothesize that using a visual-aid protocol depicting different level of residue coverage can help respondents to provide more accurate responses than simply answering an open question. Visual-aid protocols are relatively easy to integrate into existing agricultural surveys and have the potential to overcome language or educational barriers, which may be covariate with estimation error. Second, the field of human vision may limit the accurate measurement of residue coverage over a large area of land. Thus, data collected through ground observations may only be accurate for a small portion of a specific plot that is assessed by human eyes. Consequently, this paper explore the use of

\footnotetext{
1 For statistical institutes, a relevant measure of adoption would be based on the criterion that the method used is at least reliable to determine the presence of a minimum $30 \%$ field coverage.
} 


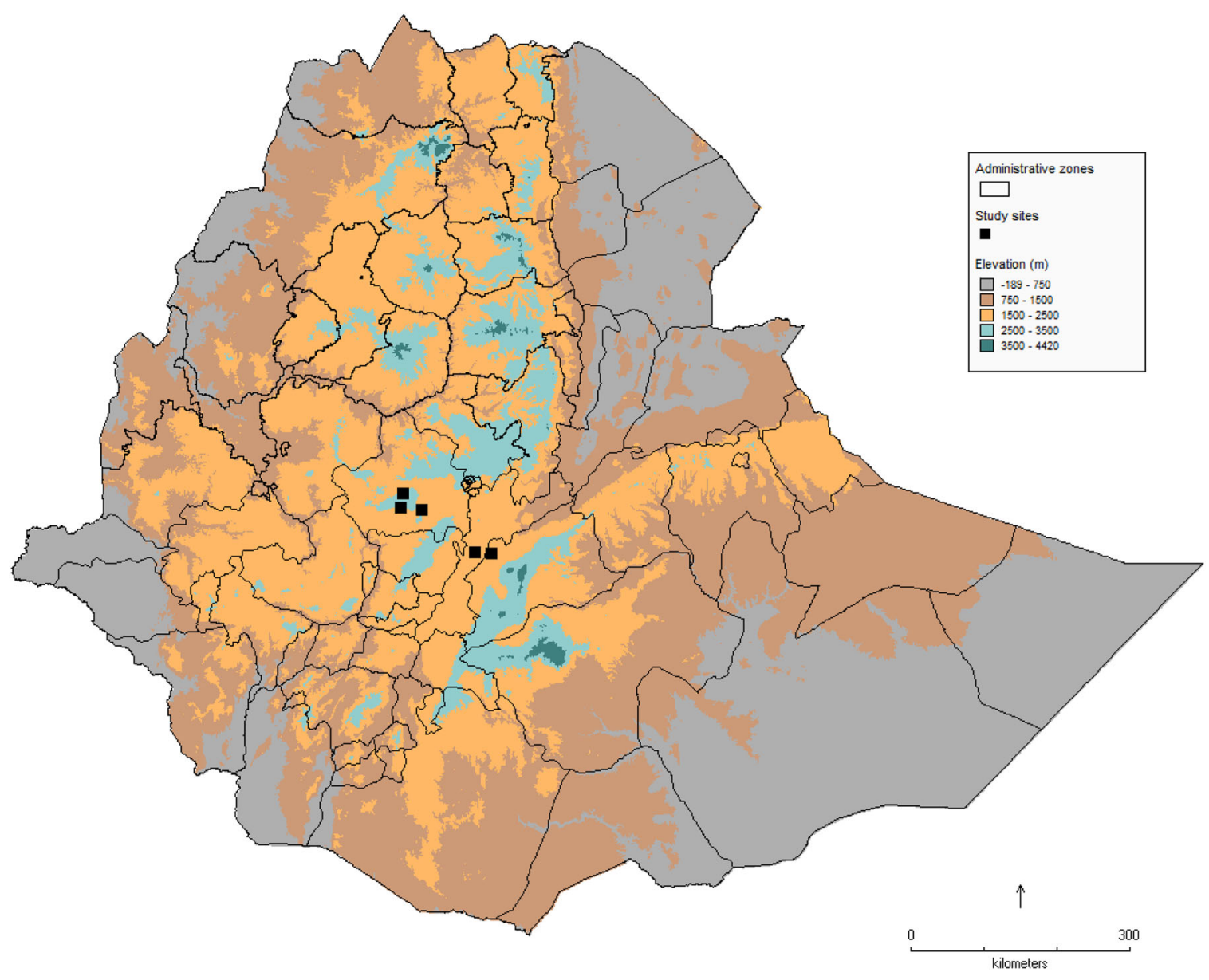

Fig. 1 Map of Ethiopia showing the location of study sites in the East and West Shewa zones

aerial data, collected through drones and satellites. We hypothesize that aerial measurement methods perform better than methods based on human observation from the ground.

\section{Data and Methods}

\section{Survey Experiment}

The data capture experiment was implemented in five enumeration areas located in the sub-humid areas of East and West Shewa zones in Ethiopia (Fig. 1). The sub-humid agro-ecological areas of Ethiopia are relatively more suitable for the adoption of crop residue cover (Alemu et al. 2006; Tesfaye et al. 2015). In each enumeration area, 12 panel households from the Ethiopian Socio-Economic Survey were interviewed. ${ }^{2}$ In addition, 28 households were randomly selected to participate in the experiment.

\footnotetext{
${ }^{2}$ ESS is the Living Standards Measurement Study-Integrated Surveys on Agriculture (LSMS-ISA) program in Ethiopia.
}

Data collection took place in December 2015 in East Shewa and February 2016 in West Shewa. Informed written consent was obtained from each household and enumerators were closely supervised, ensuring the collection of highquality data. This resulted in a total sample of 197 households and 314 plots.

Table 1 provides descriptive statistics of the sample, which is representative of the five enumeration areas. Smallscale agriculture is widely practiced, with an average farm size of 1.2 ha. The sample is well balanced between crop residue types (wheat, maize, barley, teff) and soil types (vertisol, leptosol, luvisol, cambisol). Half of the farmers in the sample indicated that they had received information by extension services on the use of crop residues in the past. However, almost all farmers (95\%) in the sample use crop residues as animal feed, while $19 \%$ use residues for firewood and 5\% use residues for construction purposes ${ }^{3}$. Communal grazing of cropland outside the cropping season is a common practice, limiting the farmers' ability to completely control the fate of the crop residues on their plots.

\footnotetext{
${ }^{3}$ Farmers generally have multiple uses for crop residues.
} 
Table 1 Background statistics of the sampled households and fields

\begin{tabular}{ll} 
Household characteristics & \\
Household size & 5.6 \\
Sex of the head (male) & 49.4 \\
Age of the head (years) & 46.2 \\
Years of education of the head & 3.3 \\
Herd size (in Tropical Livestock Units (TLU)) & 2.8 \\
Total farm size (ha) & 1.2 \\
Field characteristics & \\
Field size (m $\left.{ }^{2}\right)$ & 2139 \\
Distance from household (m) & 433 \\
Barley residues (\%) & 22.0 \\
Maize residues (\%) & 28.0 \\
Teff residues (\%) & 19.1 \\
Wheat residues (\%) & 30.9 \\
Cambisol (\%) & 14.6 \\
Leptosol (\%) & 25.5 \\
Luvisol (\%) & 20.7 \\
Vertisol (\%) & 39.2 \\
\hline
\end{tabular}

Seven methods of crop residue coverage measurement are used in this paper and summarized in Table 2-the LT method and six alternative methods for comparison (M1-6). The survey questionnaire included two modules. Module-1 took place at the interviewee's home. Tablets equipped with the Open Data Kit application were utilized to collect data on socio-demographic characteristics and farming activities of the household. Fields of maize, wheat, barley and teff were eligible for the experiment and a maximum of two fields were randomly selected for the second module of the survey. ${ }^{4}$ The home-administered Module-1 was then used to collect the respondent estimation (M1) based on recall. This method, applied for example in Jaleta et al. (2015), closely replicates the conditions of typical agricultural household surveys.

The visual-aid protocol (M3, see Fig. A.1) was also presented to respondents at home, who were then invited to identify the photo most closely matching the current state of their eligible plots. To avoid potential bias, the order of M1/ M3 and M2/M4 questions was randomized.

Module-2 was completed by the enumerator at the plot, accompanied by the farmer, and methods M2 and M4 were completed in a randomized sequence. Finally, the plots were georeferenced ${ }^{5}$ and a LT was used. Highly applied in agronomy and ecology, the line-transect method is

\footnotetext{
${ }^{4}$ In Afaan Oromo language, maize residue are named "Agada", wheat and barley residues "Galabaa" and teff residues "Qaranii".

5 GPS coordinates for each corner of the plot was taken with a $<5 \mathrm{~m}$ accuracy.
}

considered a reliable way to determine residue cover (Laflen et al. 1981; Shelton et al. 1995; Kline 2000). A $30 \mathrm{~m}$ rope with markings at $1 \mathrm{~m}$ intervals was operated by enumerators. First, the LT was laid diagonally on the field's corners. Then, enumerators were trained to look straight down from directly above each mark and count the number of marks on the rope that intersect over a piece of residue. The operation was repeated at the four corners of the field. These four measures were then averaged to obtain an estimate of residue cover for the entire field. To confirm the reliability of the LT, measures of 20 fields were taken at a 2 month interval. In all cases, the expected pattern of reduction in crop residue cover is observed, with a reduction of $25 \%$ on average.

\section{Drone Image Processing}

Low-cost drones (Phantom 2+) were used to capture aerial pictures of the surveyed fields. Since there is a relationship between the altitude required to capture a full image of a field and image resolution, a bias could arise in comparing full field pictures that have different resolutions. Thus, we made the choice to use a unique resolution for all drone aerial pictures and the drones were piloted to take a picture at a $7.5 \mathrm{~m}$ altitude from the field center. At this altitude, the image covers approximately $80 \mathrm{~m}^{2}$ and provides a resolution of $0.27 \mathrm{~cm} / \mathrm{pixel}$.

Image processing techniques are a fast and convenient method for assessing residues on the ground (Woebbeck et al. 1995; Asadi and Jafari 2011). The method of analysis consists of extracting Red-Green-Blue (RGB) components and apply an algorithm to segment the residues from the soil in the images. Image segmentation was performed with the Fiji software (Schindelin et al. 2012) and the steps followed to produce the estimate are presented in Fig. 2. First, a color balance transformation was applied to enhance contrasts between colors. Second, the RGB components of the image were extracted from the full-color image. In order to achieve the segmentation of the residues from the soil, the $2 * \mathrm{G}-\mathrm{R}-\mathrm{B}$ formula was applied as a third step (Asadi and Jafari 2011). The transformation resulted in a binary image with white pixels representing residues and black pixels representing the soil (Fig. 2c). Finally, the percentage of crop residue coverage was determined by dividing the white pixels by the total of pixels from the image.

\section{Remote Sensing Analysis}

Research on mapping conservation practices using remote sensing methods has flourished in recent years. Several indices such as the cellulose absorption index, the lignincellulose absorption index, the shortwave IR normalized residue index (SINDRI) or the normalized difference tillage 
Table 2 Survey experiment methods

\begin{tabular}{llll}
\hline Method & Measurement & Description & \\
\hline LT & Line-transect & Average of four measures taken at the cardinal points of the field & 314 \\
M1 & Interviewee estimation & Percentage estimation, away from field & 314 \\
M2 & Enumerator estimation & Percentage estimation, visiting the field & 314 \\
M3 & Interviewee visual-aid & Identification among six pictures, away from field & 314 \\
M4 & Enumerator visual-aid & Identification among six pictures, visiting the field & 314 \\
M5 & Drone image processing & Field picture taken by a drone at a 7.5 m altitude $(0.27 \mathrm{~cm} /$ pixel resolution) used to segment & 182 \\
& & RGB components & 251 \\
M6 & Remote sensing & Landsat 8 Thematic Mapper satellite imagery Multispectral (30 m/pixel resolution) used to & \\
& & compute a Normalized Difference Tillage Index (NDTI). & \\
\hline
\end{tabular}

Fig. 2 Residue segmentation image processing: a original field picture taken by a drone at a 7.5 meter altitude, b color balance transformation, $\mathbf{c}$ extraction of RGB components and $\mathbf{d}$ segmentation result after application of the $2 * \mathrm{G}-\mathrm{R}-\mathrm{B}$ formula. Soil is represented in black pixels while residues are in white pixels a)

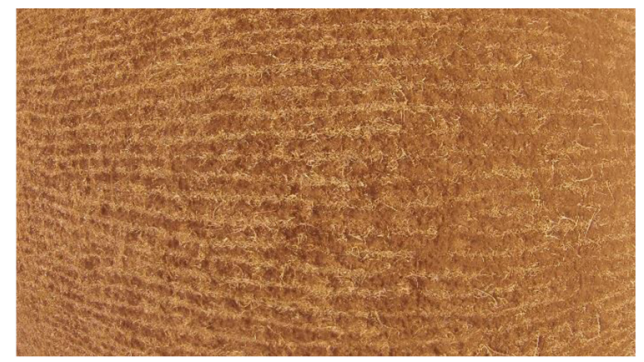

c)

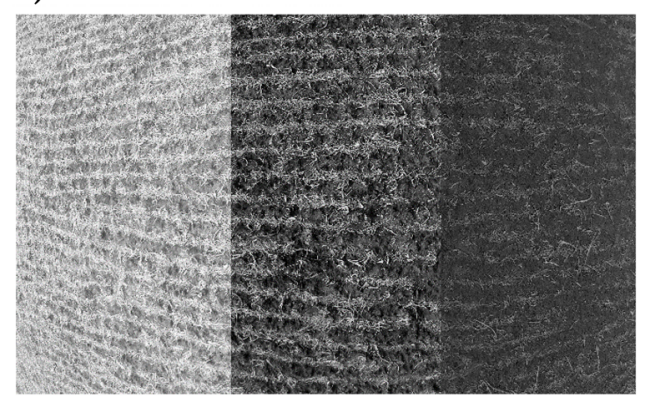

b)

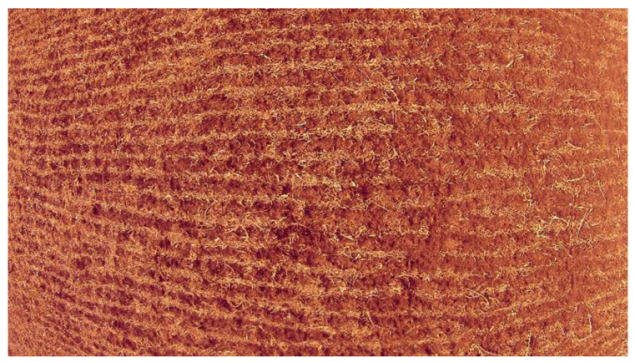

d)

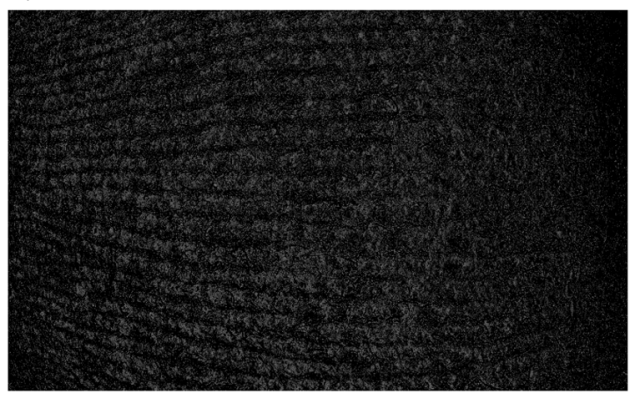

index (NDTI) have been applied with encouraging results (Nagler et al. 2003; Serbin et al. 2009a, b; Daughtry et al. 2010; Zheng et al. 2014). The calculation of these indices rely on various sensor types and bandwidths. Landsat 8 Thematic Mapper (TM) satellite images were chosen because the images are freely available and the satellite has a 16 days revisit interval. Landsat TM images were used to calculate the NDTI, considered to be the best Landsat-based tillage index (Serbin et al. 2009a, b; Zheng et al. 2014).

After survey completion, two archived full scenes of Landsat 8 TM satellite imagery were acquired from the United States Geological Survey's Earth Explorer imagery search and delivery website. The two full scenes of interest were identified based on their complete coverage of the provided field location coordinates identified above, as well as the plot-based data collection dates associated with each field location (7 December 2015 for East Shewa and 16
February 2016 for West Shewa). Thus, all measurement methods collected during the survey (LT and M1 to M5) refer to the same time as remote sensing measurements. Following the contribution of van Deventer et al. (1997), the NDTI was calculated using the formula:

$$
\text { NDTI }=\text { SWIR1 }- \text { SWIR2/SWIR1 + SWIR2 }
$$

The index was then scaled from 0 to 100 for comparison with other measures. The index was not calibrated.

\section{Data Analysis}

Reference results from the line-transect method are used to compare all other methods. Of particular interest in this study is how different measurement methods perform in estimating quantitative vs. categorical measures of crop residue cover. First, the analysis is implemented using 
Fig. 3 Boxplots of mean crop residue coverage $(\%)$ between the benchmark (LT) and the six alternative measurement methods

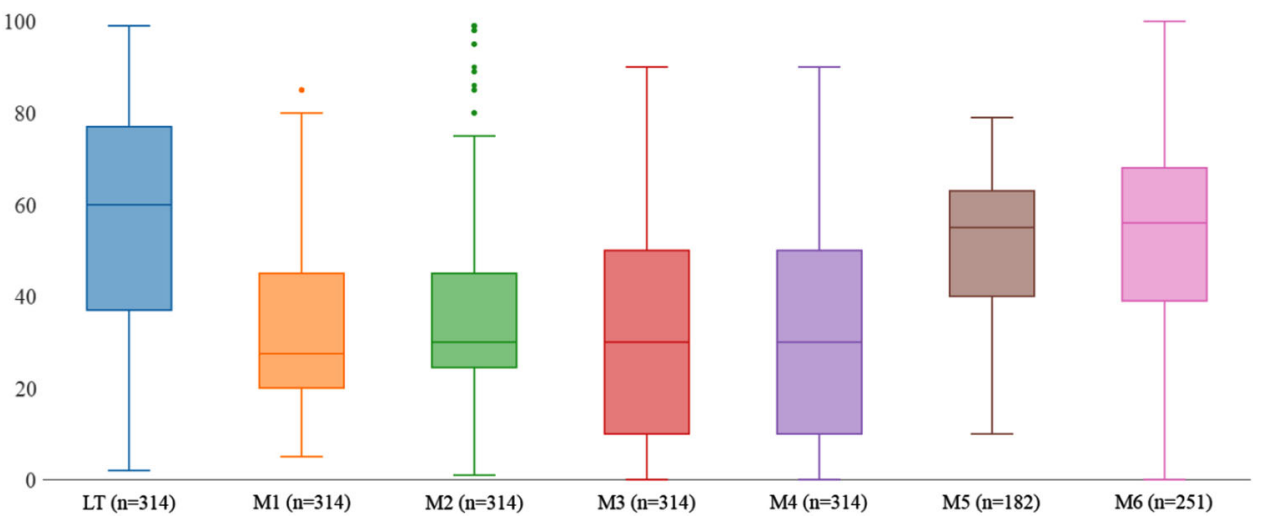

quantitative data. Boxplots are used to explore the average estimates provided by each measurement method. To further study the distribution of each measurement method and understand how well they perform along the entire distribution, we employ correlation coefficients and scatterplots. Second, we compare the six methods in terms of how aggregate binary (i.e., yes / no) adoption estimates for the plots meeting the sufficient condition of $30 \%$ residue coverage. Following FAO's AQUASTAT, three categories of adopters: $30-60,60-90$, and $>90 \%$ ground cover are also distinguished in the analysis for each method. Finally, we use a series of linear probability regressions to estimate measurement errors of adoption of a minimum $30 \%$ residue coverage. The dependent variable is equal to 1 if there is a measurement error (false negative or false positive) or 0 otherwise. A set of covariates expected to influence measurement methods are used as independent variables. Statistical analysis was performed in $\mathrm{R}$ version 3.3.1 ( $\mathrm{R}$ Development Core Team 2015).

\section{Results}

\section{Distribution of Measurement Methods}

In Fig. 3, we present box plots for the different measurement methods. We observe that the line-transect reference measure shows a full range of possible values for crop residue coverage, with a distribution ranging from 0 to 100 . The median residue cover measured by the LT is $60 \%$.

When considering the median, a major gap exists between the line-transect benchmark and all four surveybased methods (M1-4). The interviewee estimation method (M1) underestimates residue cover by 30 percent points. Having the enumerator visit the plot (M2) only performs slightly better than M1 (+2.5 percent). Surprisingly, collecting data through a visual-aid protocol (M3) does not seem to provide better estimates of the mean distribution than methods based on un-aided responses (M1). The
Table 3 Spearman's rho correlations between crop residues coverage measurement methods

\begin{tabular}{llllllll}
\hline & LT & M1 & M2 & M3 & M4 & M5 & M6 \\
\hline LT & 1 & & & & & & \\
M1 & 0.60 & 1 & & & & & \\
M2 & 0.73 & 0.68 & 1 & & & & \\
M3 & 0.59 & 0.76 & 0.55 & 1 & & & \\
M4 & 0.76 & 0.62 & 0.75 & 0.6 & 1 & & \\
M5 & -0.25 & -0.32 & -0.16 & -0.26 & -0.28 & 1 & \\
M6 & 0.57 & 0.42 & 0.39 & 0.42 & 0.47 & $0.09 *$ & 1
\end{tabular}

All correlations significant at the $p<0.001$ level at the exception of *, not significant

median distribution of the drone method (M5) appears closer from the line-transect method ( -5 percent). However, data collected with this method appear relatively concentrated in terms of overall distribution, suggesting a lack of precision at low and high ends of the spectrum of residue cover. The remote sensing method (M6) has a median and overall distribution that appear to best match the LT benchmark.

\section{How Well Did The Measures Correlate?}

Although the overall distribution of each method across the sample provides an interesting first check, we are interested in the correlations between methods at the plot level (Table 3). The interviewee and enumerator visual-aids (M2 and M4) showed the highest coefficients with the linetransect benchmark (0.73 and 0.76). Correlations were lower for interviewee and enumerator estimations $(0.60$ and $0.57)$, as well as for the remote sensing method (0.57). Counter-intuitively, the drone image processing method has a negative coefficient of -0.25 .

We also observe correlations between interviewee's perceptions (M1 and M3) and enumerator's perceptions (M2 and M4). This demonstrates coherence between respondent's answers, whether it is the interviewee or the enumerator. 
A - Interviewee estimation (M1)

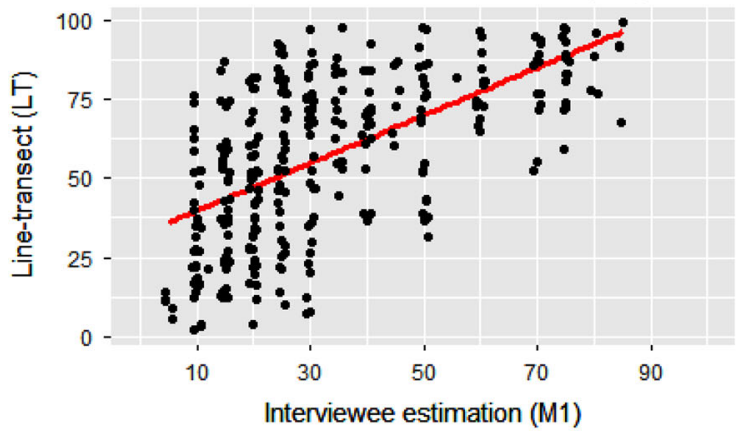

C - Interviewee visual-aid (M3)

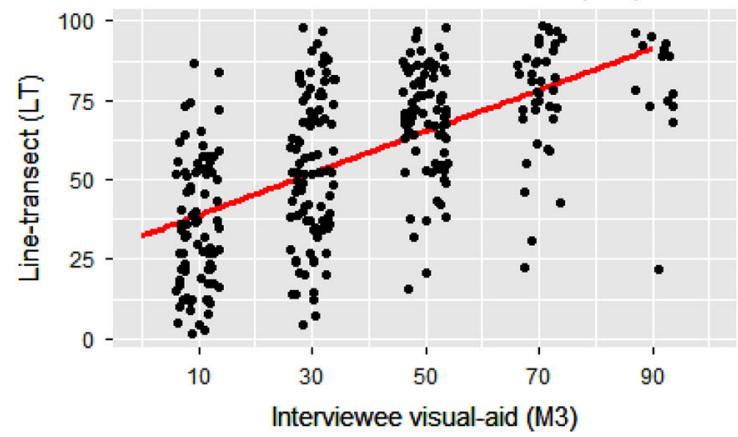

E - Drone image processing (M5)

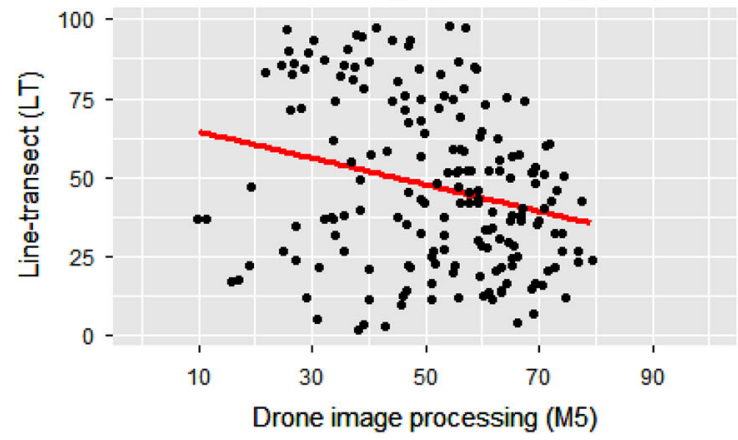

B - Enumerator estimation (M2)

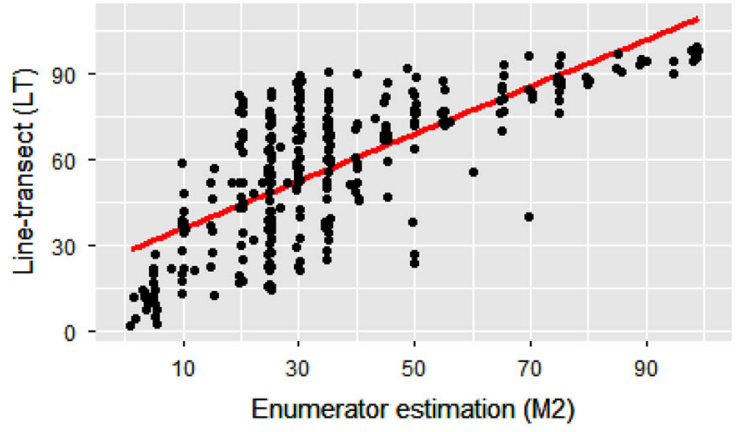

D - Enumerator visual-aid (M4)

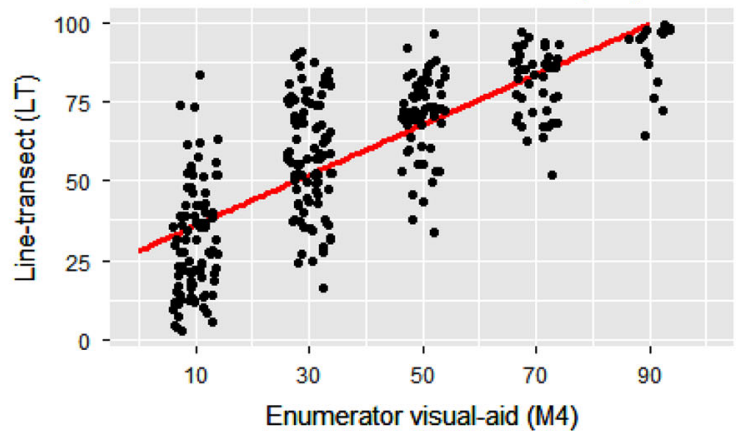

F - Remote sensing (M6)

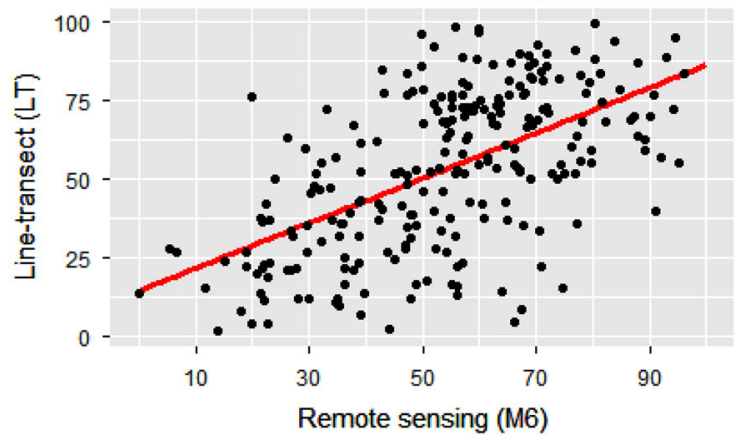

Fig. 4 Scatterplots of the six alternative measurement methods against the LT benchmark

Scatter plots of the six alternative measurement methods are plotted against the LT benchmark in Fig. 4. The red line indicates the linear fit. The underestimation of residue cover by methods M1 to M4 is confirmed at the plot level. The interviewee estimation shows under-reporting for all levels of coverage. A high level of measurement errors in the $10-30 \%$ range appears particularly problematic. This pattern is also observed in the $20-35 \%$ range in the case of enumerator estimation. However, M2 appear less likely to under-report plots with a high level of residue. Compared to the respondent visual-aid method (M3), measures obtained by enumerators (M4) have more scattered values at the $30 \%$ cover and beyond. The negative correlation of M5 does not seem to follow a consistent pattern. However, we observe that plots that are scattered along the $45^{\circ}$ line are more often vertisols and have maize residues. These two field characteristics are likely to facilitate the segmentation process of soil and residues. The remote sensing method (M6) tends to slightly underestimate low residue coverages while slightly overestimate fields with higher amounts of residues.

\section{How does Measurement Error Affect Adoption Estimates, by Method?}

In this section, we compare how adoption estimates may vary among crop residue coverage measurement methods. To what extent does the measures classify plots similarly? Figure 5 shows that all methods perform differently when 


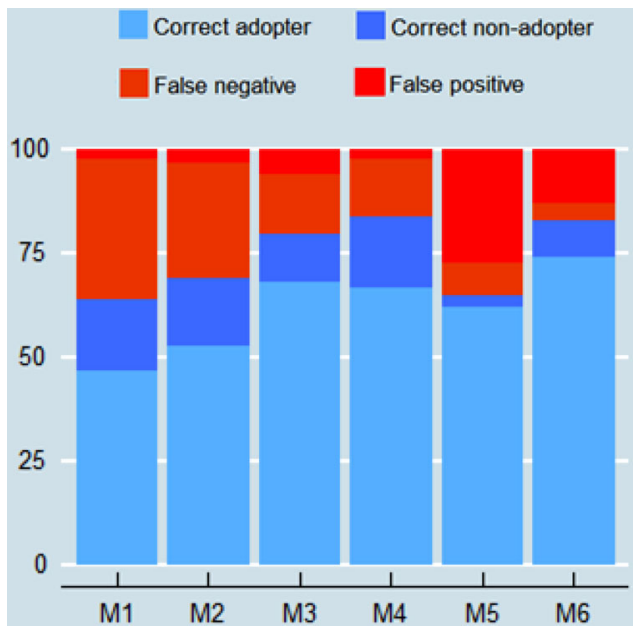

Fig. 5 Adoption false reporting of a minimum 30\% crop residue cover

using a categorical threshold. At the $30 \%$ threshold, the highest accuracy rates are provided by enumerator with visual-aid (84\%), the remote sensing method $(83 \%)$ and interviewee with visual-aid (80\%). Visual-aid methods have a higher level of false negative while remote sensing have a majority of false positive. While the remote sensing method was not as strongly correlated in continuous quantitative analysis as other methods, the categorical comparison delivers a different picture.

Next, we analyzed the extent to which each method overor under- reports adoption across the FAO AQUASTAT categories of 30-60, 60-90, and $>90 \%$ ground cover. The most consistent message that comes from Fig. 6 is that none of the measurement methods succeeds in collecting highly accurate data on a categorical scale. Despite the fact that M4 and M6 performed relatively better (Figs. 6a, b), there are still substantial measurement errors. Concerning the identification of a $>90 \%$ cover, we note that even though $4 \%$ of plots were classified as such by the line-transect, none of the alternative measurement methods was actually able to correctly classify these fields.

\section{Determinants of Measurement Errors}

As a final analysis, the determinants of measurement errors for each method are estimated. Since a 30\% minimum coverage is regarded as a threshold by conservation agriculture principles, the dependent variable is a binary outcome equal to 1 if there is a measurement error (false negative or false positive) or 0 otherwise (correct adopter or correct non-adopter). Linear probability models are used to estimate the likelihood of measurement error for each method. The models include various potential determinants of measurement errors, related to household and plot characteristics.
Household characteristics are likely to influence surveybased methods relying on the respondent's self-reporting. It is expected that the ability to estimate crop residue cover may decline with age of the respondent and that education may also improve self-reporting accuracy, especially in the case of percentage estimations. Having participated on a training in crop residue is included as an explanatory variable as trained respondents may already be familiar with crop residue management principles. In other contexts, farm size has been associated with the quality of interviewee's responses (Kondylis et al. 2015) and this variable was also included. Herd size (in Tropical Livestock Units) and the number of mobile phones in the household are used as proxies of household wealth. Since distance to the plot may decrease the frequency of visits from the interviewee, it is also hypothesized that distance to the plot could be significant in determining measurement errors.

Several plot characteristics were also included as covariates. It is recognized in the remote sensing literature that landscape components greatly impact the ability of microwave signals to detect crop residues (McNairn et al. 2001; Zheng et al. 2014). We therefore control for residue type, soil type and the amount of rocks (subjectively assessed by enumerators)—characteristics that may affect measurement errors of all methods. Field size and slope are included on the grounds that large and flat fields may result in larger measurement errors for survey-based methods and smaller errors for aerial-based methods.

The modeling results are presented in Table 4. Among survey-based methods, the strongest correlate of measurement error is field size (highly significant in 3 out of the 4 methods). This confirms the intuition that human perceptions could limit accurate residue coverage estimation. Field slope significantly decreases the likelihood of measurement errors among the M1 and M4 methods, which is consistent with the idea that more sloped fields provide a more favorable angle for someone to view the entire field and accurately estimate residue cover. The positive effect of distance on measurement error is also intuitive; compared with closer plots, more distant plots may not be visited as often or receive the same intensity of management attention. However, contrary to our expectations, aerial methods do not perform better on larger fields.

We found almost no impact of household characteristics on interviewee's answers accuracy. Consistent with Kondylis et al. (2015), farm size is the only parameter to be significantly associated with measurement errors. Surprisingly, years of education does not affect the accuracy of answers. We were unable to control for differences in enumerator's abilities in M2 and M4 owing to very small number of enumerators used so several unobserved factors may thus bias the results. 


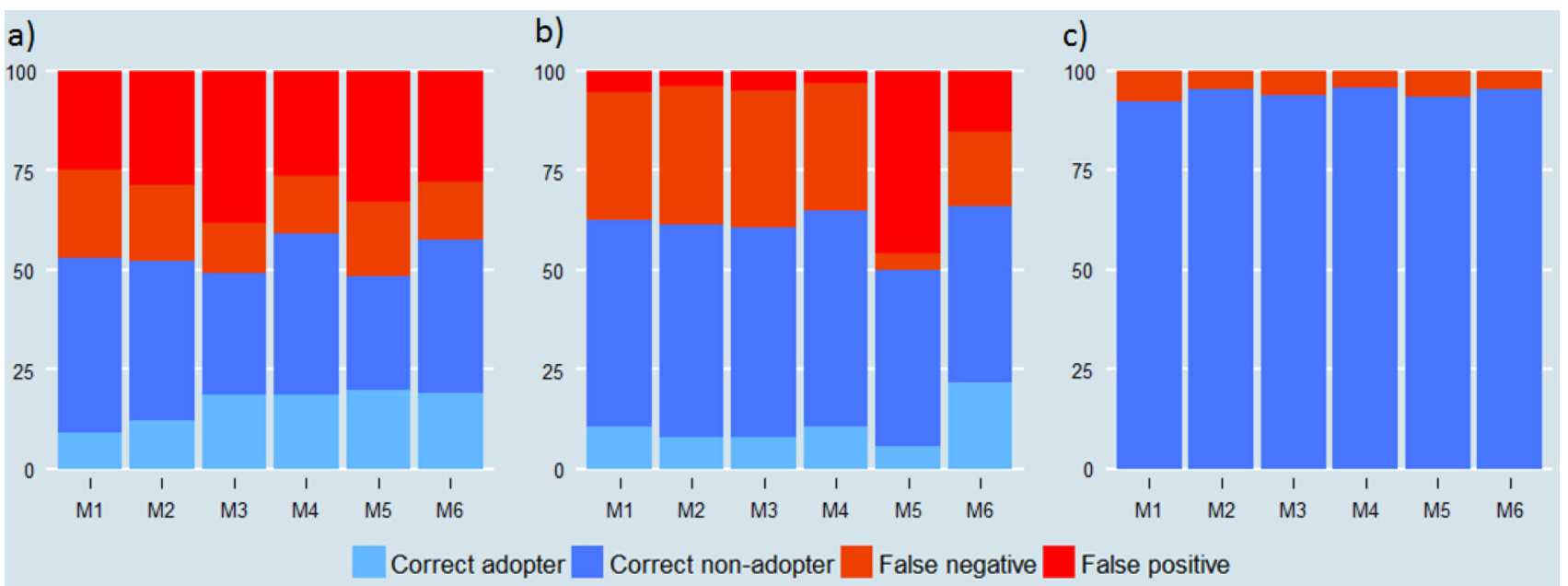

Fig. 6 Adoption false reporting of a a 30-60\% crop residue cover, b 60-90\% crop residue cover and $\mathbf{c}>90 \%$ crop residue cover by method of data collection

Crop residue and soil type also affect measurement errors of survey-based and aerial-based methods. The accuracy of enumerators' answers was lower, with more errors reported, in the case of maize and wheat residue (M3 and M4). However, wheat residues were better captured with the drone image processing method, while maize residues are associated with greater measurement errors using the remote-sensing method (M6). Luvisol (red) and vertisol (black) soil types allow respondents and enumerators to better distinguish residue cover (M1 and M4). The opposite is true for aerial-based methods where luvisol soil types increase the likelihood of measurement errors with the drone image processing method; and vertisols soils results in larger errors with the remote sensing method. This result is likely due to the higher moisture content of clay, dark soils, which may have affected spectral reflectance.

\section{Discussion and Conclusion}

Conservation agriculture has received considerable attention among scholars and policy makers in recent years. However, empirical evidence of large scale adoption and impact has remained scarce and considerable variation exists in adoption estimates (Andersson and D'Souza 2014; Stevenson et al. 2014). Despite the important implications for policy and resource allocation, very few studies have examined the accuracy of crop residue coverage-a key element of conservation agriculture.

In this article, the primary goal is to advance the discussion by presenting results of a methodological validation exercise in which six alternative methods of crop residue coverage measurement were tested among the same sample of rural households in Ethiopia, and compared against a LT benchmark. This article attempts to fill an academic and policy demand through an examination of low-cost methods for capturing field crop residue coverage information in a continuous and categorical form.

What stands out from the results is that survey-based methods tend to underestimate crop residue coverage across fields and this pattern was prevalent among interviewee's responses as well as enumerators' observations. This finding could be explained by a context of communal grazing where respondents tend to think that field residue cover is lower than it actually is. However, this explanation is not convincing for enumerators, where this result suggests inherent limits to human perceptions. Thus the methods that are often employed by National Statistical Offices would be misleading in measuring soil conservation practices. The measurement error is more serious when estimating percentages.

Despite the presence of measurement errors in all alternative methods, this research has delivered a clear ranking of measurement options. The visual-aid method yields the most accurate estimates of the true distribution as well as adoption of a minimum $30 \%$ cover. Thus, from low-cost alternative methods of data collection, results support a wider use of visual-aid protocols as an alternative to selfreported percentage cover estimations. The visual-aid protocol employed in this experiment is presented in Appendix A. It is also noteworthy that none of the alternative measurement methods were able to estimate FAO's AQUASTAT categories of 30-60, 60-90, and $>90 \%$ ground cover. Thus, the reporting of these more detailed statistics would call for more exploration.

Concerning aerial-based methods, our attempts were constrained by the necessity to identify low-cost and easy to implement solutions. This should be kept in mind, and certainly weighs on the accuracy of the results that were obtained. In contrast with results from Woebbeck et al. (1995) and Asadi and Jafari (2011) where field images were 
Table 4 Linear probability models of the factors affecting the probability of false reporting adoption (minimum 30\% coverage)

\begin{tabular}{|c|c|c|c|c|c|c|}
\hline & M1 & M2 & M3 & M4 & M5 & M6 \\
\hline \multicolumn{7}{|l|}{ Household characteristics } \\
\hline Sex of the head $(\operatorname{Ref}=$ Male $)$ & -0.07 & & 0.00 & & & \\
\hline Age of the head & 0.00 & & 0.00 & & & \\
\hline Years of education & 0.01 & & 0.01 & & & \\
\hline $\begin{array}{l}\text { Training on crop residue } \\
\text { management }\end{array}$ & -0.06 & & 0.05 & & & \\
\hline Total farm size & $0.03 * *$ & & 0.00 & & & \\
\hline Herd size (in TLU) & 0.00 & & -0.01 & & & \\
\hline Number of mobile phones & 0.01 & & 0.03 & & & \\
\hline Distance from the field & $0.00 *$ & & $0.00 * *$ & & & \\
\hline \multicolumn{7}{|l|}{ Field characteristics } \\
\hline Field size & $0.00 * * *$ & 0.00 & $0.00 * * *$ & $0.00 * *$ & 0.00 & $0.00^{*}$ \\
\hline Barley residues & -0.07 & -0.03 & 0.03 & -0.05 & 0.10 & -0.07 \\
\hline Maize residues & 0.02 & 0.04 & $0.3 * * * *$ & $0.15^{* *}$ & 0.00 & $0.3 * * *$ \\
\hline Wheat residues & 0.11 & 0.10 & $0.13 *$ & $0.11 *$ & $-0.26 * * *$ & -0.04 \\
\hline Cambisol soil type & $-0.21 * *$ & -0.12 & -0.03 & -0.08 & 0.28 & 0.1 \\
\hline Luvisol soil type & $-0.36 * * *$ & -0.11 & -0.12 & $-0.14 * *$ & $0.25^{*}$ & 0.08 \\
\hline Vertisol soil type & -0.08 & -0.05 & -0.07 & $-0.16^{* * *}$ & -0.10 & $0.24 * * *$ \\
\hline$>20 \%$ rocks & -0.04 & 0.04 & 0.02 & 0.03 & 0.02 & -0.05 \\
\hline Slight slope & $-0.14 *$ & -0.11 & -0.07 & $-0.14 * * *$ & 0.14 & -0.04 \\
\hline Steep slope & -0.08 & -0.03 & -0.17 & $-0.23 * *$ & & -0.05 \\
\hline Intercept & 0.22 & $0.33 * * *$ & 0.07 & $0.19 * *$ & $0.46 * * *$ & 0.00 \\
\hline$N$ & 314 & 314 & 314 & 314 & 182 & 251 \\
\hline Adjusted $R^{2}$ & 0.17 & 0.01 & 0.17 & 0.15 & 0.07 & 0.25 \\
\hline
\end{tabular}

$*, * *, * * *$ Statistically significant at the $0.1,0.05$, and 0.01 level respectively obtained at a distance of about $2.4 \mathrm{~m}$ height from the ground, under controlled conditions, results from the drone image processing method in this experiment were not satisfactory. In comparison with ground field images, the use of aerial images taken by drones certainly introduces additional sources of errors. An examination of aerial images taken at different altitudes has revealed modifications in the color of soil components. This suggests that the sensitivity of camera sensors to the prevailing lighting conditions may explain the inability of the segmentation algorithm to discriminate the crop residues from the soil. Given their potential to monitor adoption of agricultural technologies, research on the use of drones should be pursued; more sophisticated technologies may allow for higher accuracy of image-processing methods. ${ }^{6}$

\footnotetext{
${ }^{6}$ Piloting and picture-taking are fairly easy procedures that can be performed by enumerators with few hours of training. During the survey, the camera apparels ceased to function, possibly due to the effect of dust on electronic components. It should also be noted that given the recent introduction of these apparels, obtaining legal authorizations in Ethiopia, in the absence of a legal framework, was a tedious process.
}

In binary outcome estimates of whether a plot meets a minimum $30 \%$ residue cover threshold, the remote sensing method performed well, with an $83 \%$ accuracy rate. Given the fact that Landsat 8 images have a fairly coarse resolution sensor ( $30 \mathrm{~m} / \mathrm{pixel})$ and that the NDTI index was not calibrated, this result is very encouraging. Indeed, the relatively large pixel size of Landsat 8 and the relatively small field sizes utilized in the study may generate "mixedpixel" situations where only portions of a $30 \mathrm{~m}$ pixel actually fall over a given field. Therefore, some of the NDTI calculations could be reporting measures of residue that may be outside the field boundaries. Using a higher resolution sensor for this analysis would help alleviate this issue, though some "mixed-pixels" are almost always inevitable with remotely sensed imagery from a space platform. In addition, results suggest that the NDTI could gain in accuracy by research focusing on the effect of field variations (crop residue type, moisture, soil type/color) on spectral reflectance. The recent availability of high quality, freely available satellite data (Landsat 8, Sentinel-1), as well as the prospect of multi-sensors combining multispectral and hyperspectral data gives plenty of room for optimism. 
Some limitations of this study should be acknowledged. First, the sample is representative of a few enumeration areas only. Since measurement errors are likely to be different in different socio-economic and agro-ecological contexts, the replication of this methodological experiment in different setting should be encouraged. Second, some aspects of crop residue cover that are important for research could not be explored in this paper. These include the depth of the residue cover as well as the timing of data collection. While farmers in the area are using minimum tillage, uncontrolled grazing limit their ability to implement conservation agriculture. Future studies should take into consideration residue cover measurement over a wider period of time, possibly by looking at several agricultural seasons. In addition, soil moisture and residue moisture contentparticularly important for remote sensing estimation-could not be explored in this paper.

However, results reported here can potentially serve as guidance for survey practitioners and have implications for future household surveys. Survey-based analyses of adoption relying on self-reported estimation should be taken with caution and we advocate a wider use of visual-aid protocols (see Fig. A.1) for collecting survey-based data on natural resource management practices. Although aerial-based methods provide several promising research paths for the improvement of agricultural data, this experiment suggests that crop residue cover can be measured on the ground. We hope that these results will be taken up in future questionnaire design by National Statistical Institutes and researchers working on adoption and measurement of impact of conservation agriculture.

Acknowledgements This work was supported by the Bill \& Melinda Gates Foundation through the Strengthening Impact Assessment in the CGIAR (SIAC) program [grant number OPP1009472]. We would like to thank Theodor Friedrich, Josef Kienzle and the German Conservation Tillage Association for supplying images for the visual aid protocol as well as Biratu Yigezu and the Central Statistical Agency of Ethiopia for support. This research has benefited from the comments of Karen Macours, Timothy Kelley, and Moti Jaleta. The authors would like to thank the two anonymous reviewers for their helpful comments.

\section{Compliance with Ethical Standards}

Conflict of Interest The authors declare that they have no competing interests.

Open Access This article is distributed under the terms of the Creative Commons Attribution 4.0 International License (http://crea tivecommons.org/licenses/by/4.0/), which permits unrestricted use, distribution, and reproduction in any medium, provided you give appropriate credit to the original author(s) and the source, provide a link to the Creative Commons license, and indicate if changes were made.

\section{References}

Alemu T, Chairatanayuth P, Vijchulata P, Tudsri S (2006) Production and utilization of crop residues in three agro ecological zones of eastern Shoa Zone, Ethiopia. Kasetsart J-Nat Sci 40:643-651

Andersson JA, D'Souza S (2014) From adoption claims to understanding farmers and contexts: a literature review of Conservation agriculture (CA) adoption among smallholder farmers in southern Africa. Agric Ecosyst Environ 187:116-132. doi:10.1016/j.agee. 2013.08.008

Asadi V, Jafari A (2011) Precise measurement of residue cover by means of image processing techniques. Int J Nat Eng Sci 5:53-56

Carletto C, Savastano S, Zezza A (2013) Fact or artifact: the impact of measurement errors on the farm size-productivity relationship. J Dev Econ 103:254-261. doi:10.1016/j.jdeveco.2013.03.004

Corbeels, M, Sakyi, RK, Kühne, RF, Whitbread A (2014) Meta-Analysis of Crop Responses to Conservation Agriculture in sub-Saharan Africa. CCAFS Report No. 12. CGIAR Research Program on Climate Change, Agriculture and Food Security, Copenhagen.

Daughtry CST, Serbin G, Reeves JB, Doraiswamy PC, Hunt ER (2010) Spectral reflectance of wheat residue during decomposition and remotely sensed estimates of residue cover. Remote Sens 2:416-431. doi:10.3390/rs2020416

Erenstein O, Sayre K, Wall P, Hellin J, Dixon J (2012) Conservation agriculture in maize- and wheat-based systems in the (Sub)tropics: lessons from adaptation initiatives in South Asia, Mexico, and Southern. Africa J Sustain Agr 36:180-206. doi:10.1080/ 10440046.2011.620230

FAO (2016) AQUASTAT Main Database, Food and Agriculture Organization of the United Nations (FAO). Website accessed on [6 December 2015]

Jaleta M, Kassie M, Erenstein O (2015) Determinants of maize stover utilization as feed, fuel and soil amendment in mixed croplivestock systems, Ethiopia. Agric Syst 134:17-23. doi:10.1016/j. agsy.2014.08.010

Kassam A, Friedrich T, Shaxson F, Pretty J (2009) The spread of Conservation agriculture: justification, sustainability and uptake. Int J Agric Sustain 7:292-320. doi:10.3763/ijas.2009.0477

Kline R (2000) Estimating crop residue cover for soil erosion control. Soil fact sheet. Ministry of Agriculture and Food, British Columbia

Kondylis F, Mueller V, Zhu S (2015) Measuring agricultural knowledge and adoption. Agric Econ 46:449-462. doi:10.1111/agec.12173

Laflen JM, Amemiya M, Hintz EA (1981) Measuring crop residue cover. J Soil Water Conserv 36(6):341-343

McNairn H, Duguay C, Boisvert J, Huffman E, Brisco B (2001) Defining the sensitivity of multi-frequency and multi-polarized radar backscatter to post-harvest crop residue. Can J Remote Sens 27:247-263. doi:10.1080/07038992.2001.10854941

Nagler PL, Inoue Y, Glenn EP, Russ AL, Daughtry CST (2003) Cellulose absorption index (CAI) to quantify mixed soil-plant litter scenes. Remote Sens Environ 87:310-325. doi:10.1016/j. rse.2003.06.001

OECD (2001) Environmental indicators for agriculture: Methods and results. Agriculture and Food Vol. 3, Paris, pp 389-391

Pittelkow CM, Liang X, Linquist BA, van Groenigen KJ, Lee J, Lundy ME, van Gestel N, Six J, Venterea RT, van Kessel C (2015) Productivity limits and potentials of the principles of conservation agriculture. Nature 517:365-367. doi:10.1038/nature13809

R Development Core Team (2015) R: a language and environment for statistical computing. R Foundation for Statistical Computing, Vienna

Renkow M, Byerlee D (2010) The impacts of CGIAR research: a review of recent evidence. Food Policy 35:391-402. doi:10.1016/ j.foodpol.2010.04.006 
Serbin G, Daughtry CST, Hunt ER, Brown DJ, McCarty GW (2009a) Effect of soil spectral properties on remote sensing of crop residue cover. Soil Sci Soc Am J 73:1545. doi:10.2136/sssaj2008. 0311

Serbin G, Hunt ER, Daughtry CST, McCarty GW, Doraiswamy PC (2009b) An improved ASTER index for remote sensing of crop residue. Remote Sens 1:971-991. doi:10.3390/rs1040971

Shelton DP, Dickey EC, Kachman SD, Fairbanks KT (1995) Corn residue cover on the soil surface after planting for various tillage and planting systems. J Soil Water Conserv 50: 399-404

Stevenson JR, Serraj R, Cassman KG (2014) Evaluating conservation agriculture for small-scale farmers in sub-Saharan Africa. Agric Ecosyst Environ 187:1-10. doi:10.1016/j.agee. 2014.01.018

Schindelin J, Arganda-Carreras I, Frise E et al. (2012) Fiji: an opensource platform for biological-image analysis. Nat Methods 9:676-682. doi:10.1038/nmeth.2019

Tesfaye K, Jaleta M, Jena P, Mutenje M (2015) Identifying potential recommendation domains for Conservation agriculture in
Ethiopia, Kenya, and Malawi. Environ Manage 55:330-346. doi:10.1007/s00267-014-0386-8

Thierfelder C, Wall PC (2012) Effects of conservation agriculture on soil quality and productivity in contrasting agro-ecological environments of Zimbabwe. Soil Use Manage 28:209-220. doi:10.1111/j.1475-2743.2012.00406.x

van Deventer AP, Ward AD, Gowda PH, Lyon JG (1997) Using thematic mapper data to identify contrasting soil plains and tillage practices. Photogramm Eng Remote Sens 63:87-93

Woebbeck DM, Meyer GE, Von Bargen K, Mortensen DA (1995) Color indices for weed identification under various soil, residue and lighting conditions. Trans ASAE 38:259-269

Zezza A, Federighi G, Kalilou AA, Hiernaux P (2016) Milking the data: measuring milk off-take in extensive livestock systems. Experimental evidence from Niger. Food Policy 59:174-186. doi:10.1016/j.foodpol.2016.01.005

Zheng B, Campbell JB, Serbin G, Galbraith JM (2014) Remote sensing of crop residue and tillage practices: present capabilities and future prospects. Soil Tillage Res 138:26-34. doi:10.1016/j.still. 2013.12.009 